\title{
Optimal Reciprocal Reinsurance under GlueVaR Distortion Risk Measures
}

\author{
Yuxia Huang, Chuancun Yin \\ School of Statistics, Qufu Normal University, Qufu, China \\ Email: ccyin@qfnu.edu.cn
}

How to cite this paper: Huang, Y.X. and Yin, C.C. (2019) Optimal Reciprocal Reinsurance under GlueVaR Distortion Risk Measures. Journal of Mathematical Finance, 9, 11-24.

https://doi.org/10.4236/jmf.2019.91002

Received: December 16, 2018

Accepted: January 14, 2019

Published: January 17, 2019

Copyright $\odot 2019$ by author(s) and Scientific Research Publishing Inc. This work is licensed under the Creative Commons Attribution International License (CC BY 4.0).

http://creativecommons.org/licenses/by/4.0/

\section{(c) (i) Open Access}

\begin{abstract}
This article investigates the optimal reciprocal reinsurance strategies when the risk is measured by a general risk measure, namely the GlueVaR distortion risk measures, which can be expressed as a linear combination of two tail value at risk (TVaR) and one value at risk $(\mathrm{VaR})$ risk measures. When we consider the reciprocal reinsurance, the linear combination of three risk measures can be difficult to deal with. In order to overcome difficulties, we give a new form of the GlueVaR distortion risk measures. This paper not only derives the necessary and sufficient condition that guarantees the optimality of marginal indemnification functions (MIF), but also obtains explicit solutions of the optimal reinsurance design. This method is easy to understand and can be simplified calculation. To further illustrate the applicability of our results, we give a numerical example.
\end{abstract}

\section{Keywords}

Distortion Risk Measure, VaR, TVaR, GlueVaR, Marginal Indemnification Function (MIF), Optimal Reciprocal Reinsurance

\section{Introduction}

Reinsurance is an effective risk management tool for the insurer to transfer part of its risk to the reinsurer. Let $X$ be the original loss, if the insurer cedes a part of loss $f(X)$ ( $f$ is called the ceded loss function, or indemnification function) to the reinsurer and pays reinsurance premium $\delta_{f}(X)$, then the insurer's total liability $T_{I_{f}}(X)$ contains two parts: one is the retained loss risk $I_{f}(X)=X-f(X)$ and the other is the reinsurance premium $\delta_{f}(X)$, that is

$$
T_{I_{f}}(X)=X-f(X)+\delta_{f}(X) .
$$

The reinsurer's total liability $T_{R_{f}}(X)$ also contains two parts: one is the 
ceded loss risk $R_{f}(X)=f(X)$ and the other is the received reinsurance premium $\delta_{f}(X)$, that is

$$
T_{R_{f}}(X)=f(X)-\delta_{f}(X) .
$$

For any $\lambda \in[0,1]$, we define total risks $T_{f}(X)$ in the presence of an insurer and a reinsurer as

$$
T_{f}(X)=\lambda T_{I_{f}}(X)+(1-\lambda) T_{R_{f}}(X) .
$$

Due to the development and application of risk measures in finance and insurance, many workers formulate the optimal reinsurance problem with Value at Risk (VaR) and Tail Value at Risk (TVaR). [1] proposed two optimization criterion that minimize total loss of the insurer by the Value at Risk (VaR) and the Conditional Tail Expectation (CTE). [2] showed that quota-share and stop-loss reinsurance are optimal when they studied a class of increasing convex ceded loss functions by VaR and CTE under the expected value principle. Many works extended the fundamental results, for example, [3]-[15]. [16] extended the conclusion of [15] to the general convex risk measure that satisfied regular invariance. Recently, there has a surge of interest in more generally distortion risk measures. [17] discussed the general model of the distortion risk measure and assumed that the distortion function is piecewise convex or concave. [18] studied the general model with distortion risk measures under general reinsurance premium principles. [19] expended the model of [18] under the cost-benefit framework. [20] studied the optimal reinsurance model of [18] without the premium constraint by a marginal indemnification function (MIF) formula. [21] studied the optimal reinsurance with premium constraint by combining the MIF formula and the Lagrangian dual method. [22] and [23] studied the optimal reinsurance with constraints under the distortion risk measure.

$\mathrm{VaR}$ has been adopted as the standard tool for assessing the risks and calculating the capital requirements in finance and insurance, however, it has two drawbacks in financial industry. One is that the capital requirements can be underestimated and the underestimated may be aggravated when heavy tail losses are incorrectly modeled by mild tail distribution. The second one is that the VaR may fail the subadditivity. Though TVaR has no these two disadvantages of VaR, it has not been widely accepted by practitioners in finance and insurance. In order to overcome this weakness, [24] proposed a new family of risk measures, namely GlueVaR distortion risk measures. We take different definitions of VaR from [24], therefore, a new definition of GlueVaR has been given in this paper.

Optimal reinsurance from an insurer's viewpoint or from a reinsurer's viewpoint has been studied for a long time in the literatures. However, as two parties of a reinsurance contract, there has a conflict of interests between an insurer and a reinsurer. The optimal reinsurance policy from one party's perspective may not be optimal for another party. Therefore, we consider a reciprocal reinsurance. Motivated by [21] and [24], we want to study the optimal reciprocal rein- 
surance strategy under GlueVaR distortion risk measures with MIF formula.

The rest of this paper is organized as follows. In Section 2, we give some notations and proposal a reciprocal reinsurance model. In Section 3, we derive the sufficient conditions that guarantee the existence of a reinsurance contract. In Section 4 , we obtain the specific expression of optimal reinsurance. Section 5 concludes this paper.

\section{The Model}

\subsection{Preliminaries and Notations}

Definition 2.1. (Distortion risk measure or distorted expectation) A distortion function is a non-decreasing function $g:[0,1] \rightarrow[0,1]$ such that $g(0)=0$ and $g(1)=1$. The distortion risk measure or distorted expectation of the random variable $X$ associated with distortion function $g$, notation $\varrho_{g}(X)$, is defined as

$$
\varrho_{g}(X)=\int_{-\infty}^{0}\left[g\left(S_{X}(x)\right)-1\right] \mathrm{d} x+\int_{0}^{\infty} g\left(S_{X}(x)\right) \mathrm{d} x .
$$

The most well-known examples of distortion risk measures are the VaR and $\mathrm{TVaR}$, if we define the distortion functions, respectively, as follows

$$
g_{\alpha}(x)=\mathbb{I}_{\{x>\alpha\}}
$$

and

$$
g_{\beta}(x)=\frac{x}{\beta} \mathbb{I}_{\{x \leq \beta\}}+\mathbb{I}_{\{x>\beta\}},
$$

then the distorted expectation $\varrho_{g}(X)$ can be equivalently expressed as

$$
\operatorname{VaR}_{\alpha}(X)=\inf \{x: P(X>x) \leq \alpha\}=S_{X}^{-1}(\alpha)
$$

and

$$
\operatorname{TVaR}_{\alpha}(X)=\frac{1}{\alpha} \int_{0}^{\alpha} \operatorname{VaR}_{q}(X) \mathrm{d} q=\frac{1}{\alpha} \int_{0}^{\alpha} S_{X}^{-1}(q) \mathrm{d} q .
$$

Definition 2.2. (GlueVaR distortion risk measure) Given the confidence levels $1-\alpha$ and $1-\beta$, when the distortion function for GlueVaR is specified to the following function

$$
g_{\beta, \alpha}^{h_{1}, h_{2}}(x)= \begin{cases}\frac{h_{1}}{\beta} \times x, & x \in[0, \beta], \\ h_{1}+\frac{h_{2}-h_{1}}{\alpha-\beta} \times(x-\beta), & x \in[\beta, \alpha], \\ 1, & x \in[\alpha, 1],\end{cases}
$$

with $\alpha, \beta \in[0,1], \alpha>\beta, h_{1} \in[0,1]$, and $h_{2} \in\left[h_{1}, 1\right]$, then the corresponding distortion risk measure $\varrho_{g}$ is the GlueVaR distortion risk measure, which is denoted by GlueVaR ${ }_{\beta, \alpha}^{h_{1}, h_{2}}(X)$.

Remark 2.1. If the following notation is used, 


$$
\left\{\begin{array}{l}
\omega_{1}=h_{1}-\frac{h_{2}-h_{1}}{\alpha-\beta} \times \beta, \\
\omega_{2}=\frac{h_{2}-h_{1}}{\alpha-\beta} \times \alpha, \\
\omega_{3}=1-h_{2},
\end{array}\right.
$$

then the distortion function $g_{\beta, \alpha}^{h_{1}, h_{2}}(x)$ in (2.6) may be rewritten as

$$
g_{\beta, \alpha}^{h_{1}, h_{2}}(x)=\omega_{1} g_{T, \beta}(x)+\omega_{2} g_{T, \alpha}(x)+\omega_{3} g_{V, \alpha}(x),
$$

where $g_{T, \beta}(x), g_{T, \alpha}(x)$ and $g_{V, \alpha}(x)$ are the distortion functions corresponding to the $\operatorname{TVaR}_{\beta}(X), \operatorname{TVaR}_{\alpha}(X)$ and $\operatorname{VaR}_{\alpha}(X)$, respectively. Therefore, GlueVaR is a risk measure that can be expressed as a linear combination of three risk measures as follows,

$$
\operatorname{GlueVaR}_{\beta, \alpha}^{h_{1}, h_{2}}(X)=\omega_{1} \operatorname{TVaR}_{\beta}(X)+\omega_{2} \operatorname{TVaR}_{\alpha}(X)+\omega_{3} \operatorname{VaR}_{\alpha}(X),
$$

where $\omega_{i} \in[0,1]$ for $i=1,2,3$, and $\omega_{1}+\omega_{2}+\omega_{3}=1$.

Example 2.1. Assume that initial risk $X$ follows an exponential distribution with parameter 0.001, then $\operatorname{VaR}_{\alpha}(X)=-1000 \ln (\alpha)$,

$\operatorname{TVaR}_{\alpha}(X)=-1000 \ln (\alpha)+1000$. When $\omega_{1}=0.2, \omega_{2}=0.3$ and $\omega_{3}=0.5$, the values of VaR, TVaR and GlueVaR at different confidence levels are calculated in Table 1.

Given $\alpha$ and $\beta$, the values in Table 1 indicate that GlueVaR is more conservative than $\operatorname{VaR}$. Note that $\operatorname{VaR}_{\alpha}(X) \leq \operatorname{GlueVaR}_{\beta, \alpha}^{h_{1}, h_{2}}(X)$, which means that GlueVaR may overcome the VaR's shortage of underestimating risks. On the other hand, GlueVaR is not, unlike TVaR, overly conservative. It seems clear that GlueVaR, a new risk measure based on distortion functions, can be valuable in the scope of finance and insurance.

Definition 2.3. (Marginal indemnification function) (See [[20], Definition 2]) For any indemnification function $f(X)$, the associated marginal indemnification is a function $h \in[0,1]$ such that

$$
f(x)=\int_{0}^{x} h(t) \mathrm{d} t, x \geq 0 .
$$

\subsection{Model Set-Up}

Based on the notations of the preceding subsection, we will introduce a reciprocal reinsurance model to study the optimal strategy which considers the interests of both an insurer and a reinsurer.

Problem 1 (Optimization model of a reciprocal reinsurance)

$$
\operatorname{GlueVaR}_{\beta, \alpha}^{h_{1}, h_{2}}\left(T_{f^{*}}(X)\right)=\min _{f \in \mathcal{F}} \operatorname{GlueVaR}_{\beta, \alpha}^{h_{1}, h_{2}}\left(T_{f}(X)\right),
$$

where $\mathcal{F}=\left\{f(x): f(x)\right.$ and $I_{f}(x)$ are non-decreasing and $\left.f(x)=\int_{0}^{x} h(t) \mathrm{d} t, \quad 0 \leq h(t) \leq 1\right\}$.

Our objective is to find the optimal ceded loss function $f^{*}(X)$ and to characterize the corresponding $\operatorname{GlueVaR}_{\beta, \alpha}^{h_{1}, h_{2}}\left(f^{*}(X)\right)$. 
Table 1. VaR, TVaR and GlueVaR of initial risk X.

\begin{tabular}{cccccc}
\hline$\beta$ & 0.01 & 0.03 & 0.05 & 0.07 & 0.09 \\
\hline$\alpha$ & 0.02 & 0.04 & 0.06 & 0.08 & 0.10 \\
$\mathrm{TVaR}_{\beta}(X)$ & 5605.2 & 4506.6 & 3995.7 & 3659.3 & 3407.9 \\
$\mathrm{TVaR}_{\alpha}(X)$ & 4912.0 & 4218.9 & 3813.4 & 3525.7 & 3302.6 \\
$\operatorname{VaR}_{\alpha}(X)$ & 3912.0 & 3218.9 & 2813.4 & 2525.7 & 2302.6 \\
$\operatorname{GlueVaR}_{\beta, \alpha}^{h_{1}, l_{2}}(X)$ & 4550.6 & 3776.4 & 3349.9 & 3052.4 & 2823.7 \\
\hline
\end{tabular}

\section{Existence of Optimal Reinsurance Strategy}

Lemma 3.1 For any ceded loss functions $f(X)$, $\operatorname{GlueVaR}_{\beta, \alpha}^{h_{1}, h_{2}}(f(X))$ can be expressed as

$$
\begin{aligned}
& \text { GlueVaR } h_{\beta, \alpha}^{h_{1}, h_{2}}(f(X)) \\
& =\int_{0}^{\infty}\left[\omega_{1} g_{T, \beta}\left(S_{X}(x)\right)+\omega_{2} g_{T, \alpha}\left(S_{X}(x)\right)+\omega_{3} g_{V, \alpha}\left(S_{X}(x)\right)\right] h(x) \mathrm{d} x,
\end{aligned}
$$

where $\omega_{i} \in[0,1]$ for $i=1,2,3$, and $\omega_{1}+\omega_{2}+\omega_{3}=1$.

Proof. As proved in Lemma 2.1 of Zhuang et al. (2016), for any distortion function $g$,

$$
\varrho_{g}(f(X))=\int_{0}^{\infty} g\left[S_{X}(t)\right] \mathrm{d} f(t) .
$$

Obviously, GlueVaR $\mathrm{h}_{\beta, \alpha}^{h_{1}, h_{2}}(f(X))$ may be rewritten as (3.1).

Lemma 3.2 For any $\lambda \in[0,1]$ and ceded loss function $f(X)$, total risks $T_{f}(X)$ can be expressed as

$\operatorname{GlueVaR}_{\beta, \alpha}^{h_{1}, h_{2}}\left(T_{f}(X)\right)=\lambda \operatorname{GlueVaR}_{\beta, \alpha}^{h_{1}, h_{2}}(X)+(1-2 \lambda) \int_{0}^{\infty} \varphi\left(S_{X}(x)\right) h(x) \mathrm{d} x,(3.2)$ where

$$
\varphi\left(S_{X}(x)\right)=\omega_{1} g_{T, \beta}\left(S_{X}(x)\right)+\omega_{2} g_{T, \alpha}\left(S_{X}(x)\right)+\omega_{3} g_{V, \alpha}\left(S_{X}(x)\right)-(1+\rho) S_{X}(x) .
$$

Proof. From definitions of $T_{I_{f}}(X)$ and $T_{R_{f}}(X), T_{f}(X)$ can be rewritten as

$$
T_{f}(X)=\lambda X+(1-2 \lambda)\left[f(X)-\delta_{f}(X)\right] .
$$

By the comonotonic additivity of the distortion risk measures, total risks $T_{f}(X)$ under the GlueVaR distortion risk measures can be expressed as

$$
\begin{aligned}
\operatorname{GlueVaR}_{\beta, \alpha}^{h_{1}, h_{2}}\left(T_{f}(X)\right)= & \lambda \operatorname{GlueVaR}_{\beta, \alpha}^{h_{1}, h_{2}}(X)+(1-2 \lambda) \operatorname{GlueVaR}_{\beta, \alpha}^{h_{1}, h_{2}}(f(X)) \\
& -(1-2 \lambda) \delta_{f}(X) .
\end{aligned}
$$

Based on the fact that

$$
\delta_{f}(X)=(1+\rho) E(f(X))=(1+\rho) \int_{0}^{\infty} S_{X}(x) h(x) d x,
$$

with the expressions (3.1), (3.4) and (3.5), we get

$$
\begin{aligned}
& \operatorname{GlueVaR}_{\beta, \alpha}^{h_{1}, h_{2}}\left(T_{f}(X)\right) \\
& =\lambda \operatorname{GlueVaR}_{\beta, \alpha}^{h_{1}, h_{2}}(X)+(1-2 \lambda) \int_{0}^{\infty}\left[\omega_{1} g_{T, \beta}\left(S_{X}(x)\right)\right. \\
& \left.+\omega_{2} g_{T, \alpha}\left(S_{X}(x)\right)+\omega_{3} g_{V, \alpha}\left(S_{X}(x)\right)-(1+\rho) S_{X}(x)\right] h(x) \mathrm{d} x .
\end{aligned}
$$


Lemma 3.3 Let $h^{*}$ be the optimal marginal indemnification function, then it satisfies

$$
\begin{aligned}
& \min _{f \in \mathcal{F}} \operatorname{GlueVaR}_{\beta, \alpha}^{h_{1}, h_{2}}\left(T_{f}(X)\right) \\
& =\lambda \operatorname{GlueVaR}_{\beta, \alpha}^{h_{1}, h_{2}}(X)+(1-2 \lambda) \int_{0}^{\infty} \varphi\left(S_{X}(x)\right) h^{*}(x) \mathrm{d} x .
\end{aligned}
$$

Suppose that $f^{*}(x)=\int_{0}^{x} h^{*}(z) \mathrm{d} z$ for $x \in[0, \infty)$. Then $h^{*}$ solves (3.6) if and only if $f^{*}$ solves (2.11).

Proof. This follows from the same arguments used in the proof to Proposition 2.1 of Zhuang et al. (2016).

Theorem 3.1 For $\lambda \in[0,1], h^{*}(x)$ solves 3.6 if and only if it satisfies the followings.

1). If $0 \leq \lambda<\frac{1}{2}$, then

$$
h^{*}(x)= \begin{cases}1, & \varphi\left(S_{X}(x)\right)<0, \\ \xi \in[0,1], & \varphi\left(S_{X}(x)\right)=0, \\ 0, & \varphi\left(S_{X}(x)\right)>0 .\end{cases}
$$

2). If $\lambda=\frac{1}{2}$, then

$$
h^{*}(x)=\xi \in[0,1] .
$$

3). If $\frac{1}{2}<\lambda \leq 1$, then

$$
h^{*}(x)= \begin{cases}0, & \varphi\left(S_{X}(x)\right)<0, \\ \xi \in[0,1], & \varphi\left(S_{X}(x)\right)=0, \\ 1, & \varphi\left(S_{X}(x)\right)>0 .\end{cases}
$$

Proof. Note that minimizing GlueVaR $h_{\beta, \alpha}^{h_{1}, h_{2}}\left(T_{f}(X)\right)$ is equivalent to minimizing $(1-2 \lambda) \int_{0}^{\infty} \varphi\left(S_{X}(x)\right) h(x) \mathrm{d} x$ of (3.2). In the next, we will prove the results from three cases.

1). For the cases $0 \leq \lambda<\frac{1}{2}, 1-2 \lambda>0$.

a) If $\varphi\left(S_{X}(x)\right)<0$, then the minimum $(1-2 \lambda) \int_{0}^{\infty} \varphi\left(S_{X}(x)\right) h(x) \mathrm{d} x$ is attained at $h(x)=1$.

b) If $\varphi\left(S_{X}(x)\right)=0$, then $(1-2 \lambda) \int_{0}^{\infty} \varphi\left(S_{X}(x)\right) h(x) \mathrm{d} x=0$ for any $h(x)=\xi \in[0,1]$.

c) If $\varphi\left(S_{X}(x)\right)>0$, then the minimum $(1-2 \lambda) \int_{0}^{\infty} \varphi\left(S_{X}(x)\right) h(x) \mathrm{d} x$ is attained at $h(x)=0$.

2). For the cases $\lambda=\frac{1}{2},(1-2 \lambda) \int_{0}^{\infty} \varphi\left(S_{X}(x)\right) h(x) \mathrm{d} x=0$ for any $h(x)=\xi \in[0,1]$.

3). For the cases $\frac{1}{2}<\lambda \leq 1,1-2 \lambda<0$. 
a) If $\varphi\left(S_{X}(x)\right)<0$, then the minimum $(1-2 \lambda) \int_{0}^{\infty} \varphi\left(S_{X}(x)\right) h(x) \mathrm{d} x$ is attained at $h(x)=0$.

b) If $\varphi\left(S_{X}(x)\right)=0$, then $(1-2 \lambda) \int_{0}^{\infty} \varphi\left(S_{X}(x)\right) h(x) \mathrm{d} x=0$ for any $h(x)=\xi \in[0,1]$.

c) If $\varphi\left(S_{X}(x)\right)>0$, then the minimum $(1-2 \lambda) \int_{0}^{\infty} \varphi\left(S_{X}(x)\right) h(x) \mathrm{d} x$ is attained at $h(x)=1$.

\section{Explicit Solutions}

In Section 3, we have derived the optimal marginal indemnification function $h^{*}$. It seems very concise but we can not obtain the optimal reinsurance strategy $f^{*}$ directly. In this section, we want to derive the optimal reinsurance contract $f^{*}$ bases on optimal marginal indemnification function $h^{*}$.

Let $t=S_{X}(x)$ and denote $\psi(t)=\varphi\left(S_{X}(x)\right)$, we have

$$
\psi(t)=\omega_{1} g_{T, \beta}(t)+\omega_{2} g_{T, \alpha}(t)+\omega_{3} g_{V, \alpha}(t)-(1+\rho) t,
$$

where

$$
\begin{gathered}
g_{T, \beta}(x)=\frac{x}{\beta} \mathbb{I}_{\{x \leq \beta\}}+\mathbb{I}_{\{x>\beta\}}, \\
g_{T, \alpha}(x)=\frac{x}{\alpha} \mathbb{I}_{\{x \leq \alpha\}}+\mathbb{I}_{\{x>\alpha\}}, \\
g_{V, \alpha}(x)=\mathbb{I}_{\{x>\alpha\}} .
\end{gathered}
$$

With the expression (4.1)-(4.4), $\psi(t)$ may be reexpressed as

$$
\psi(t)= \begin{cases}k_{1} t, & {[0, \beta],} \\ k_{2} t+\omega_{1}, & (\beta, \alpha], \\ k_{3} t+1, & (\alpha, 1],\end{cases}
$$

which has two positive zeros,

$$
t_{1}=\frac{\omega_{1} \alpha}{(1+\rho) \alpha-\omega_{2}}, \quad t_{2}=\frac{1}{1+\rho},
$$

where

$$
\begin{gathered}
k_{1}=\frac{\omega_{1}}{\beta}+\frac{\omega_{2}}{\alpha}-(1+\rho), \\
k_{2}=\frac{\omega_{2}}{\alpha}-(1+\rho), \\
k_{3}=-(1+\rho) .
\end{gathered}
$$

Theorem 4.1 For any ceded loss function $f(x) \in \mathcal{F}$, if $\lambda=\frac{1}{2}$, then

$$
f^{*}(x)=\xi x, \quad \xi \in[0,1] .
$$

Proof. From (2.10) and (3.8), we can derive above results easily.

Theorem 4.2 For $0 \leq \lambda<\frac{1}{2}$, and any ceded loss function $f(x) \in \mathcal{F}$, optim- 
al reinsurance contracts $f^{*}$ to Problem 1 are given as follows.

1). If $k_{1}>0$ and $k_{2} \geq 0$, then $f^{*}(x)=x \wedge S_{X}^{-1}\left(t_{2}\right)$.

2). If $k_{1}>0$ and $k_{2}<0$, then

$f^{*}(x)= \begin{cases}x \wedge S_{X}^{-1}\left(t_{2}\right), & \psi(\alpha) \geq 0, \\ x \wedge S_{X}^{-1}\left(t_{2}\right)+\left(x-S_{X}^{-1}(\alpha)\right)_{+} \wedge\left(S_{X}^{-1}\left(t_{1}\right)-S_{X}^{-1}(\alpha)\right), & \psi(\alpha)<0, \psi(\alpha+)>0 . \\ x \wedge S_{X}^{-1}\left(t_{1}\right), & \psi(\alpha)<0, \psi(\alpha+) \leq 0,\end{cases}$

3). If $k_{1}=0$, then

$f^{*}(x)= \begin{cases}x \wedge S_{X}^{-1}\left(t_{2}\right)+\left(x-S_{X}^{-1}(\alpha)\right)_{+} \wedge\left(S_{X}^{-1}(\beta)-S_{X}^{-1}(\alpha)\right)+\xi\left(x-S_{X}^{-1}(\beta)\right)_{+}, & \psi(\alpha+)>0, \\ x \wedge S_{X}^{-1}(\beta)+\xi\left(x-S_{X}^{-1}(\beta)\right)_{+}, & \psi(\alpha+) \leq 0 .\end{cases}$

4). If $k_{1}<0$, then

$$
f^{*}(x)= \begin{cases}x \wedge S_{X}^{-1}\left(t_{2}\right)+\left(x-S_{X}^{-1}(\alpha)\right)_{+}, & \psi(\alpha+)>0 \\ x, & \psi(\alpha+) \leq 0 .\end{cases}
$$

Proof. Analyse the optimal reinsurance contract with (3.7) for the case $0 \leq \lambda<\frac{1}{2}$. From (4.5)-(4.8), clearly $k_{1}>k_{2}>k_{3}$ and $k_{3}<0$. Note that $\psi(\beta)=\psi(\beta+)$, but $\psi(\alpha)<\psi(\alpha+)$, which means that $\psi(t)$ is discontinuous at the point $t=\alpha$. Therefore, we consider the followings.

1). When $k_{1}>0$, there has three cases about $k_{2}$, which are $k_{2}>0, k_{2}=0$ and $k_{2}<0$.

a) If $k_{2}>0$, then $\psi(\alpha)>0 . t_{2}$ exists since $\psi(\alpha+)>\psi(\alpha)>0$ and $\psi(1)=-\rho<0$. Note that $\psi(t)>0$ in $\left(0, t_{2}\right), \psi(t)<0$ in $\left(t_{2}, 1\right]$ as Figure 1. With the expression (3.7), we have that $h^{*}(x)=1$ for $x \in\left(0, S_{X}^{-1}\left(t_{2}\right)\right)$, $h^{*}(x)=0$ for $x \in\left(S_{X}^{-1}\left(t_{2}\right), \infty\right)$ as Figure 2, thus $f^{*}(x)=x \wedge S_{X}^{-1}\left(t_{2}\right)$.

b) If $k_{2}=0$, then $\psi(\alpha)>0$. Similar to 1$), f^{*}(x)=x \wedge S_{X}^{-1}\left(t_{2}\right)$.

c) When $k_{2}<0, \psi(\alpha)$ has three cases $\psi(\alpha)>0, \psi(\alpha)=0$ and $\psi(\alpha)<0$. Since $\psi(t)$ is discontinuous at the point $t=\alpha$, we have to consider the cases of $\psi(\alpha+)$.

i) If $\psi(\alpha) \geq 0$, then $\psi(\alpha+)>0$. Therefore, $t_{2}$ exists. $\psi(t)>0$ in $\left(0, t_{2}\right)$, $\psi(t)<0$ in $\left(t_{2}, 1\right]$. Furthermore, $h^{*}(x)=1$ for $x \in\left(0, S_{X}^{-1}\left(t_{2}\right)\right), h^{*}(x)=0$ for $x \in\left(S_{X}^{-1}\left(t_{2}\right), \infty\right)$, so $f^{*}(x)=x \wedge S_{X}^{-1}\left(t_{2}\right)$.

ii) If $\psi(\alpha)<0$, then $t_{1}$ exists. If $\psi(\alpha+)>0$, then $t_{2}$ exists since $\psi(1)<0$. Note that $\psi(t)>0$ in $\left(0, t_{1}\right)$ and $\left(\alpha+, t_{2}\right], \psi(t)<0$ in $\left(t_{1}, \alpha\right]$ and $\left(t_{2}, 1\right]$. Furthermore, $h^{*}(x)=1$ for $x \in\left(0, S_{X}^{-1}\left(t_{2}\right)\right) \cup\left(S_{X}^{-1}(\alpha), S_{X}^{-1}\left(t_{1}\right)\right)$, $h^{*}(x)=0$ for $x \in\left(S_{X}^{-1}\left(t_{2}\right), S_{X}^{-1}(\alpha)\right) \cup\left(S_{X}^{-1}\left(t_{1}\right), \infty\right)$, so $f^{*}(x)=x \wedge S_{X}^{-1}\left(t_{2}\right)+\left(x-S_{X}^{-1}(\alpha)\right)_{+} \wedge\left(S_{X}^{-1}\left(t_{1}\right)-S_{X}^{-1}(\alpha)\right)$.

iii) If $\psi(\alpha)<0$, then $t_{1}$ exists. When $\psi(\alpha+) \leq 0, \psi(t)>0$ in $\left(0, t_{1}\right)$, $\psi(t)<0$ in $\left(t_{1}, 1\right]$. Furthermore, $h^{*}(x)=1$ for $x \in\left(0, S_{X}^{-1}\left(t_{1}\right)\right), h^{*}(x)=0$ for $x \in\left(S_{X}^{-1}\left(t_{1}\right), \infty\right)$, so $f^{*}(x)=x \wedge S_{X}^{-1}\left(t_{1}\right)$.

2). When $k_{1}=0$, from (4.6) and (4.7), we obtain that $k_{2}<0$ and $\psi(\alpha)<0$. Next, we consider the cases of $\psi(\alpha+)$. 


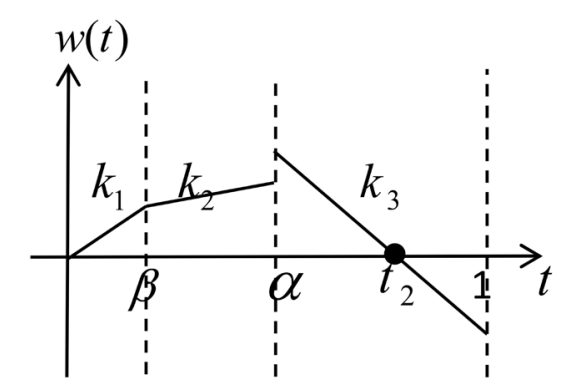

Figure 1. $k_{1}>0, k_{2}>0$.

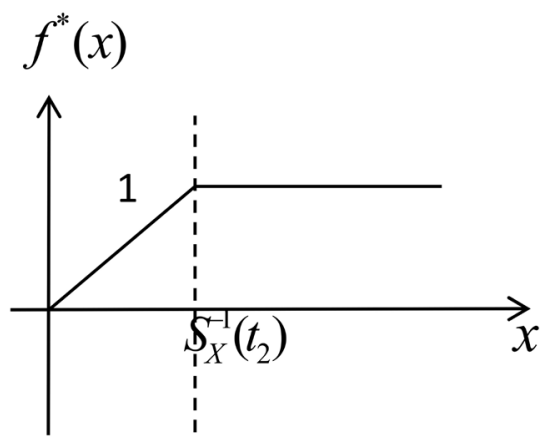

Figure 2. $0 \leq \lambda<\frac{1}{2}$.

a) When $\psi(\alpha+)>0$, we can derive that $\psi(t)>0$ in $\left(\alpha, t_{2}\right), \psi(t)=0$ in $[0, \beta]$, and $\psi(t)<0$ in $(\beta, \alpha]$ and $\left(t_{2}, 1\right]$. Furthermore, $h^{*}(x)=1$ for $x \in\left[0, S_{X}^{-1}\left(t_{2}\right)\right) \cup\left(S_{X}^{-1}(\alpha), S_{X}^{-1}(\beta)\right), h^{*}(x)=0$ for $x \in\left(S_{X}^{-1}\left(t_{2}\right), S_{X}^{-1}(\alpha)\right)$, and $h^{*}(x)=\xi$ for $x \in\left[S_{X}^{-1}(\beta), \infty\right)$. Therefore,

$f^{*}(x)=x \wedge S_{X}^{-1}\left(t_{2}\right)+\left(x-S_{X}^{-1}(\alpha)\right)_{+} \wedge\left(S_{X}^{-1}(\beta)-S_{X}^{-1}(\alpha)\right)+\xi\left(x-S_{X}^{-1}(\beta)\right)_{+}$.

b) When $\psi(\alpha+) \leq 0, \psi(t)=0$ in $[0, \beta]$ and $\psi(t)<0$ in $(\beta, 1]$.

Furthermore, $h^{*}(x)=1$ for $x \in\left[0, S_{X}^{-1}(\beta)\right)$, and $h^{*}(x)=\xi$ for $x \in\left[S_{X}^{-1}(\beta), \infty\right)$. Therefore, $f^{*}(x)=x \wedge S_{X}^{-1}(\beta)+\xi\left(x-S_{X}^{-1}(\beta)\right)_{+}$.

3). When $k_{1}<0$, note that $k_{2}<0$ and $\psi(\alpha)<0$. There has three cases for $\psi(\alpha+)$.

a) When $\psi(\alpha+)>0, \psi(t)>0$ in $\left(\alpha, t_{2}\right)$ and $\psi(t)<0$ in other cases. Furthermore, $h^{*}(x)=1$ for $x \in\left(0, S_{X}^{-1}\left(t_{2}\right)\right) \cup\left(S_{X}^{-1}(\alpha), \infty\right), h^{*}(x)=0$ for $x \in\left(S_{X}^{-1}\left(t_{2}\right), S_{X}^{-1}(\alpha)\right)$. Therefore, $f^{*}(x)=x \wedge S_{X}^{-1}\left(t_{2}\right)+\left(x-S_{X}^{-1}(\alpha)\right)_{+}$.

b) If $\psi(\alpha+) \leq 0$, then $\psi(t)<0$ in $(0,1)$. Therefore, $h^{*}(x)=1$ when $x \in(0, \infty), f^{*}(x)=x$.

Theorem 4.3 For $\frac{1}{2}<\lambda \leq 1$, and any ceded loss function $f(x) \in F$, optimal reinsurance contracts $f^{*}$ to Problem 1 are given as follows.

1). If $k_{1}>0$ and $k_{2} \geq 0$, then $f^{*}(x)=\left(x-S_{X}^{-1}\left(t_{2}\right)\right)_{+}$.

2). If $k_{1}>0$ and $k_{2}<0$, then 


$$
f^{*}(x)= \begin{cases}\left(x-S_{X}^{-1}\left(t_{2}\right)\right)_{+}, & \psi(\alpha) \geq 0, \\ \left(x-S_{X}^{-1}\left(t_{2}\right)\right)_{+} \wedge\left(S_{X}^{-1}(\alpha)-S_{X}^{-1}\left(t_{2}\right)\right)+\left(x-S_{X}^{-1}\left(t_{1}\right)\right)_{+}, & \psi(\alpha)<0, \psi(\alpha+)>0, \\ \left(x-S_{X}^{-1}\left(t_{1}\right)\right)_{+}, & \psi(\alpha)<0, \psi(\alpha+) \leq 0 .\end{cases}
$$

3). If $k_{1}=0$, then

$$
f^{*}(x)= \begin{cases}\left(x-S_{X}^{-1}\left(t_{2}\right)\right)_{+} \wedge\left(S_{X}^{-1}(\alpha)-S_{X}^{-1}\left(t_{2}\right)\right)+\xi\left(x-S_{X}^{-1}(\beta)\right)_{+}, & \psi(\alpha+)>0, \\ \xi\left(x-S_{X}^{-1}(\beta)\right)_{+}, & \psi(\alpha+) \leq 0 .\end{cases}
$$

4). If $k_{1}<0$, then

$$
f^{*}(x)= \begin{cases}\left(x-S_{X}^{-1}\left(t_{2}\right)\right)_{+} \wedge\left(S_{X}^{-1}(\alpha)-S_{X}^{-1}\left(t_{2}\right)\right), & \psi(\alpha+)>0, \\ 0, & \psi(\alpha+) \leq 0 .\end{cases}
$$

Proof. Analyse the optimal reinsurance contract with (3.9) for the case $\frac{1}{2}<\lambda \leq 1$.

1). When $k_{1}>0$, there has three cases about $k_{2}$.

a) If $k_{2}>0$, then $\psi(\alpha)>0$. Since $\psi(\alpha+)>\psi(\alpha)>0$ and $\psi(1)=-\rho<0$, then $t_{2}$ exists. Therefore, $\psi(t)>0$ in $\left(0, t_{2}\right)$ and $\psi(t)<0$ in $\left(t_{2}, 1\right]$ as Figure 1. With the expression (3.9), we have that $h^{*}(x)=0$ for $x \in\left(0, S_{X}^{-1}\left(t_{2}\right)\right)$ and $h^{*}(x)=1$ for $x \in\left(S_{X}^{-1}\left(t_{2}\right), \infty\right)$ as Figure 3 , so $f^{*}(x)=\left(x-S_{X}^{-1}\left(t_{2}\right)\right)$.

b) If $k_{2}=0$, then $\psi(\alpha)>0$. Similar to 1$), f^{*}(x)=\left(x-S_{X}^{-1}\left(t_{2}\right)\right)$.

c) When $k_{2}<0, \psi(\alpha)$ has three cases $\psi(\alpha)>0, \psi(\alpha)=0$ and $\psi(\alpha)<0$. Since $\psi(t)$ is discontinuous at the point $t=\alpha$, we have to consider the cases of $\psi(\alpha+)$.

i) If $\psi(\alpha) \geq 0$, then $\psi(\alpha+)>0, t_{2}$ exists since $\psi(1)=-\rho<0$. Note that $\psi(t)>0$ in $\left(0, t_{2}\right)$ and $\psi(t)<0$ in $\left(t_{2}, 1\right]$. Furthermore, $h^{*}(x)=0$ for $x \in\left(0, S_{X}^{-1}\left(t_{2}\right)\right), h^{*}(x)=1$ for $x \in\left(S_{X}^{-1}\left(t_{2}\right), \infty\right)$, so $f^{*}(x)=\left(x-S_{X}^{-1}\left(t_{2}\right)\right)$.

ii) If $\psi(\alpha)<0$ and $\psi(\alpha+)>0$, then $t_{1}$ and $t_{2}$ exists. Clearly $\psi(t)>0$ in $\left(0, t_{1}\right)$ and $\left(\alpha+, t_{2}\right], \psi(t)<0$ in $\left(t_{1}, \alpha\right]$ and $\left(t_{2}, 1\right]$. Furthermore, $h^{*}(x)=0$ for $x \in\left(0, S_{X}^{-1}\left(t_{2}\right)\right) \cup\left(S_{X}^{-1}(\alpha), S_{X}^{-1}\left(t_{1}\right)\right), h^{*}(x)=1$ for $x \in\left(S_{X}^{-1}\left(t_{2}\right), S_{X}^{-1}(\alpha)\right) \cup\left(S_{X}^{-1}\left(t_{1}\right), \infty\right)$, so $f^{*}(x)=\left(x-S_{X}^{-1}\left(t_{2}\right)\right)_{+} \wedge\left(S_{X}^{-1}(\alpha)-S_{X}^{-1}\left(t_{2}\right)\right)+\left(x-S_{X}^{-1}\left(t_{1}\right)\right)_{+}$.

iii) If $\psi(\alpha)<0$ and $\psi(\alpha+) \leq 0$, then $t_{1}$ exists. Clearly, $\psi(t)>0$ in $\left(0, t_{1}\right)$ and $\psi(t)<0$ in $\left(t_{1}, 1\right]$. Furthermore, $h^{*}(x)=0$ for $x \in\left(0, S_{X}^{-1}\left(t_{1}\right)\right)$, $h^{*}(x)=1$ for $x \in\left(S_{X}^{-1}\left(t_{1}\right), \infty\right)$, so $f^{*}(x)=\left(x-S_{X}^{-1}\left(t_{1}\right)\right)_{+}$.

2). When $k_{1}=0$, from (4.6) and (4.7), we obtain that $k_{2}<0$ and $\psi(\alpha)<0$. Next, we consider the cases of $\psi(\alpha+)>0$.

a) When $\psi(\alpha+)>0$, we can derive that $\psi(t)>0$ in $\left(\alpha, t_{2}\right), \psi(t)=0$ in $[0, \beta]$, and $\psi(t)<0$ in $(\beta, \alpha]$ and $\left(t_{2}, 1\right]$. Furthermore, $h^{*}(x)=0$ for $x \in\left[0, S_{X}^{-1}\left(t_{2}\right)\right) \cup\left(S_{X}^{-1}(\alpha), S_{X}^{-1}(\beta)\right), h^{*}(x)=1$ for $x \in\left(S_{X}^{-1}\left(t_{2}\right), S_{X}^{-1}(\alpha)\right)$, and $h^{*}(x)=\xi$ when $x \in\left[S_{X}^{-1}(\beta), \infty\right)$. Therefore,

$f^{*}(x)=\left(x-S_{X}^{-1}\left(t_{2}\right)\right)_{+} \wedge\left(S_{X}^{-1}(\alpha)-S_{X}^{-1}\left(t_{2}\right)\right)+\xi\left(x-S_{X}^{-1}(\beta)\right)_{+}$. 
b) When $\psi(\alpha+) \leq 0, \psi(t)=0$ in $[0, \beta]$ and $\psi(t)<0$ in $(\beta, 1]$. Furthermore, $h^{*}(x)=0$ for $x \in\left[0, S_{X}^{-1}(\beta)\right)$, and $h^{*}(x)=\xi$ for $x \in\left[S_{X}^{-1}(\beta), \infty\right)$. Therefore, $f^{*}(x)=\xi\left(x-S_{X}^{-1}(\beta)\right)$.

3). When $k_{1}<0$, note that $k_{2}<0$ and $\psi(\alpha)<0$. There has three cases for $\psi(\alpha+)$.

a) When $\psi(\alpha+)>0, \psi(t)>0$ in $\left(\alpha, t_{2}\right)$ and $\psi(t)<0$ in other cases. Furthermore, $h^{*}(x)=0$ for $x \in\left(0, S_{X}^{-1}\left(t_{2}\right)\right) \cup\left(S_{X}^{-1}(\alpha), \infty\right), h^{*}(x)=1$ for $x \in\left(S_{X}^{-1}\left(t_{2}\right), S_{X}^{-1}(\alpha)\right)$. Therefore, $f^{*}(x)=\left(x-S_{X}^{-1}\left(t_{2}\right)\right)_{+} \wedge\left(S_{X}^{-1}(\alpha)-S_{X}^{-1}\left(t_{2}\right)\right)$.

b) If $\psi(\alpha+) \leq 0$, then $\psi(t)<0$ in $(0,1)$. Therefore, $h^{*}(x)=0$ when $x \in(0, \infty), f^{*}(x)=0$.

Example 4.1. Similar to Example 2.1, we assume the risk is measured by the GlueVaR risk measures under the expectation premium principle, for $\lambda \in[0,1]$, $\xi \in[0,1], \omega_{i} \in[0,1], \quad i=1,2,3$ and $\omega_{1}+\omega_{2}+\omega_{3}=1$, optimal reinsurance contracts are given as follows.

From the reinsurer's point of view, as Case 1 in Table 2, the optimal reinsurance strategy can be in form of limited quota-share, $f^{*}(x)=x \wedge 405.47$, which means that if initial loss $X$ less than 405.47, the case that an insurer ceded all loss to a reinsurer is optimal, and if initial loss $X$ more than 405.47 , the case that an insurer ceded 405.47 to a reinsurer is optimal.

From the insurer's point of view, as Case 6 in Table 2, the optimal reinsurance strategy $f^{*}(x)=0$, which means that an insurer should retain all loss to achieve itself optimality.

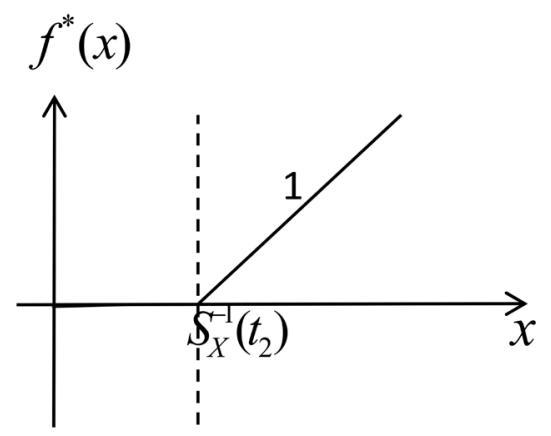

Figure 3. $\frac{1}{2}<\lambda \leq 1$.

Table 2. Optimal ceded loss function.

\begin{tabular}{ccccccccc}
\hline Case & $\alpha$ & $\beta$ & $\omega_{1}$ & $\omega_{2}$ & $\omega_{3}$ & $\lambda$ & $\rho$ & $f^{*}(x)$ \\
\hline 1 & 0.05 & 0.01 & 0.20 & 0.30 & 0.50 & 0.00 & 0.50 & $x \wedge 405.47$ \\
2 & 0.10 & 0.05 & 0.10 & 0.05 & 0.85 & 0.20 & 1.00 & $x \wedge 2995.73+\xi(x-2995.73)_{+}$ \\
3 & 0.15 & 0.10 & 0.15 & 0.10 & 0.75 & 0.40 & 2.00 & $x \wedge 1099.61+(x-1897.12)_{+}$ \\
4 & 0.20 & 0.15 & 0.40 & 0.20 & 0.40 & 0.60 & 1.50 & $(x-916.29)_{+}$ \\
5 & 0.25 & 0.20 & 0.50 & 0.20 & 0.30 & 0.80 & 2.00 & $(x-1099.61)_{+} \wedge 286.68+\xi(x-1609.44)_{+}$ \\
6 & 0.30 & 0.25 & 0.60 & 0.10 & 0.30 & 1.00 & 3.00 & 0 \\
\hline
\end{tabular}


From the perspectives of an insurer and a reinsurer, as Cases 2 - 5. Note that Cases 2 and 5 include the parameter $\xi \in[0,1]$, which means that reinsurance contracts can be different forms when the loss risk has been minimized. Case 3 means that the stop-loss after quota-share reinsurance (which is to say a stop-loss will be applied after a quota-share reinsurance) is optimal. Case 4 means that stop-loss reinsurance is optimal.

\section{Conclusion}

This article has studied the optimal reciprocal reinsurance with the GlueVaR distortion risk measures under the expected value premium principle. The Glu$\mathrm{eVaR}$ distortion risk measure is a linear combination of two TVaR and one VaR with different confidence levels, which adds the difficulty than the case of only one $\mathrm{VaR}$ or the case of only one TVaR when we derive the optimal reinsurance contract. In this paper, we have expressed GlueVaR as a linear combination of three distortion risk measures with different distortion functions. Therefore, we can use MIF formula to deal with the complex optimization problems easily. The results indicate that depending on the risk measures's level of confidence ( $\alpha$ and $\beta$ ), the safety loading $(\rho)$ for the reinsurance premium, weight $(\lambda)$ of an insurer in the reciprocal reinsurance model and the proportions $\left(\omega_{1}, \omega_{2}\right.$ and $\omega_{3}$ ) of the three risk measures in the definition of GlueVaR, the optimal reinsurance can be in the forms of quota-share, stop-loss, change-loss, or their combination, for example, stop-loss after quota-share. This paper has not considered the practical constraints, such as risk constraints or reinsurance premium constraints, which can be studied at a later time.

\section{Acknowledgements}

The author would like to thank the anonymous referees for helpful comments and suggestions, which have led to significant improvements of the present paper.

\section{Author Contributions}

These authors contributed equally to this work.

\section{Conflicts of Interest}

The authors declare no conflict of interest.

\section{Funding}

The research was supported by the National Natural Science Foundation of China (No. 11171179, 11571198).

\section{References}

[1] Cai, J. and Tan, K.S. (2007) Optimal Retention for a Stop-Loss Reinsurance under the VaR and CTE Risk Measures. Astin Bulletin, 37, 93-112. 
https://doi.org/10.2143/AST.37.1.2020800

[2] Cai, J., Tan, K.S., Weng, C. and Zhang, Y. (2008) Optimal Reinsurance under VaR and CTE Risk Measures. Insurance: Mathematics and Economics, 43, 185-196. https://doi.org/10.1016/j.insmatheco.2008.05.011

[3] Cheung, K.C. (2010) Optimal Reinsurance Revisited: A Geometric Approach. Astin Bulletin, 40, 221-239. https://doi.org/10.2143/AST.40.1.2049226

[4] Chi, Y. and Tan, K.S. (2011) Optimal Reinsurance under VaR and CVaR Risk Measures: A Simplified Approach. Astin Bulletin, 41, 487-509.

[5] Chi, Y. and Tan, K.S. (2013) Optimal Reinsurance with General Premium Principles. Insurance: Mathematics and Economics, 52, 180-189. https://doi.org/10.1016/j.insmatheco.2012.12.001

[6] Li, P., Zhou, M. and Yin, C.C. (2015) Optimal Reinsurance with Both Proportional and Fixed Costs. Statistics and Probability Letters, 106, 134-141. https://doi.org/10.1016/j.spl.2015.06.024

[7] Cai, J., Liu, H.Y. and Wang, R.D. (2017) Pareto-Optimal Reinsurance Arrangements under General Model Settings. Insurance: Mathematics and Economics, 77, 24-37. https://doi.org/10.1016/j.insmatheco.2017.08.004

[8] Chi, Y.C. and Meng, H. (2014) Optimal Reinsurance Arrangements in the Presence of Two Reinsurers. Scandinavian Actuarial Journal, 5, 424-438. https://doi.org/10.1080/03461238.2012.723638

[9] Yin, C.C. (2018) Remarks on Equality of Two Distributions under Some Partial Orders. Acta Mathematicae Applicatae Sinica, English Series, 34, 274-280.

https://doi.org/10.1007/s10255-018-0744-Z

[10] Yuen, K.C. and Yin, C.C. (2012) Asymptotic Results for Tail Probabilities of Sums of Dependent and Heavy-Tailed Random Variables. Chinese Annals of Mathematics, Series B, 33B, 557-568. https://doi.org/10.1007/s11401-012-0723-2

[11] Yin, C.C. and Zhu, D. (2016) New Class of Distortion Risk Measure and Their Tail Asymptotics with Emphasis on VaR. Journal of Financial Risk Management, 7, 12-23. https://doi.org/10.4236/jfrm.2018.71002

[12] Zhu, D. and Yin, C.C. (2018) Two Sufficient Conditions for Convex Ordering on Risk Aggregation. Abstract and Applied Analysis, 2018, Article ID: 2937895. https://doi.org/10.1155/2018/2937895

[13] Zhu, D. and Yin, C.C. (2018) Stochastic Optimal Control of Investment and Dividend Payment Model under Debt Control with Time-Inconsistency. Mathematical Problems in Engineering, 2018, Article ID: 7928953. https://doi.org/10.1155/2018/7928953

[14] Zhu, Y.Z., Chi, Y.C. and Weng, C. (2014) Multivariate Reinsurance Designs for Minimizing an Insurer's Capital Requirement. Insurance: Mathematics and Economics, 59, 144-155. https://doi.org/10.1016/j.insmatheco.2014.09.009

[15] Tan, K.S., Weng, C. and Zhang, Y. (2011) Optimality of General Reinsurance Contracts under CTE Risk Measure. Insurance: Mathematics and Economics, 49, 175-187. https://doi.org/10.1016/j.insmatheco.2011.03.002

[16] Cheung, K.C., Sung, K., Yam, S. and Yung, S. (2014) Optimal Reinsurance under General Law-Invariant Risk Measures. Scandinavian Actuarial Journal, 1, 72-91. https://doi.org/10.1080/03461238.2011.636880

[17] Zheng, Y.T. and Cui, W. (2014) Optimal Reinsurance with Premium Constraint under Distortion Risk Measures. Insurance: Mathematics and Economics, 59, 
109-120. https://doi.org/10.1016/j.insmatheco.2014.08.010

[18] Cui, W., Yang, J.P. and Wu, L. (2013) Optimal Reinsurance Minimizing the Distortion Risk Measure under General Reinsurance Premium Principles. Insurance: Mathematics and Economics, 53, 74-85.

https://doi.org/10.1016/j.insmatheco.2013.03.007

[19] Cheung, K.C. and Lo, A. (2017) Characterizations of Optimal Reinsurance Treaties: A Cost Benefit Approach. Scandinavian Actuarial Journal, 1, 1-28. https://doi.org/10.1080/03461238.2015.1054303

[20] Assa, H. (2015) On Optimal Reinsurance Policy with Distortion Risk Measures and Premiums. Insurance: Mathematics and Economics, 61, 70-75. https://doi.org/10.1016/j.insmatheco.2014.11.007

[21] Zhuang, S.C., Weng, C., Tan, K.S. and Assa, H. (2016) Marginal Indemnification Function Formulation for Optimal Reinsurance. Insurance: Mathematics and Economics, 67, 65-76. https://doi.org/10.1016/j.insmatheco.2015.12.003

[22] Lo, A. (2017) A Neyman-Pearson Perspective on Optimal Reinsurance with Constraints. Astin Bulletin, 47, 467-499. https://doi.org/10.1017/asb.2016.42

[23] Jiang, W.J., Hong, H.P. and Ren, J.D. (2017) On Pareto-Optimal Reinsurance with Constraints under Distortion Risk Measures. European Actuarial Journal, 8, 215-243. https://doi.org/10.1007/s13385-017-0163-1

[24] Belles-Sampera, J., Guillen, M. and Santolino, M. (2014) Beyond Value-at-Risk: GlueVaR Distortion Risk Measures. Risk Analysis, 34, 121-134.

https://doi.org/10.1111/risa.12080 